Espaço Aberto | Open Space

\title{
El papel de la economía informal en los proyectos agroecológicos durante el COVID-19
}

\section{The role of the informal economy in agro-ecological projects during COVID-19}

Paula Escribano*

* Universitat Autònoma de Barcelona - Barcelona, Cataluña, España paula.escribano@uab.cat https://orcid.org/0000-0002-4635-3325

Agata Hummel**

** Uniwersytet Warszawski - Varsovia, Polonia a.hummel@uw.edu.pl https://orcid.org/0000-0002-8979-602X

Claudio Milano ${ }^{\star \star \star}$

${ }^{* \star *}$ Universitat Autònoma de Barcelona - Barcelona, Cataluña, España claudio.milano@uab.cat

https://orcid.org/0000-0003-4349-367X 


\title{
Resumen
}

El 13 de marzo de 2020 España decretó el estado de alarma debido a la emergencia sanitaria por el COVID-19. En este escenario el sector agroalimentario tuvo que responder a una gran demanda en términos de producción y abastecimiento. Entre los diferentes modelos productivos dentro del sector están las pequeñas iniciativas agroecológicas que pretenden desarrollar estilos de vida campesinos por encima de la reproducción del capital. Las pequeñas iniciativas agroecológicas han tenido que adaptarse a los cambios impuestos por el estado de alarma para poder continuar con su actividad principal: la producción y distribución de alimentos a escala local. A partir de un enfoque cualitativo se reflexiona sobre los retos qué han afrontado las pequeñas iniciativas agroecológicas con las regulaciones de la vida pública y los efectos que han tenido las medidas sobre su actividad. Asimismo, la investigación se centra en cuestionar el doble papel de la economía informal como amortiguador y limitante para su propio funcionamiento. Se analiza el papel que juega la economía informal en el sector agroalimentario local en la zona periurbana de Barcelona y de Madrid.

Palabras clave: estado de alarma; COVID-19; agroecología; economía informal.

\begin{abstract}
On March 13, 2020, Spain declared a state of alarm due to the health emergency caused by COVID-19. In this scenario, the agri-food sector had to respond to a high demand in terms of production and supply. Among the different production models within the sector there are small agroecological initiatives that aim to develop peasant lifestyles beyond the reproduction of capital. The small agroecological initiatives have had to adapt to the changes imposed by the state of alarm in order to continue with their main activity: the production and distribution of food on a local scale. With a qualitative approach, the paper reflects on the challenges that small agroecological initiatives have faced with the regulations of public life and the effects that the measures have had on their activity. The research also focuses on questioning the dual role of the informal economy as both a shock absorber and a constraint. The paper analyzes the role played by the informal economy in the local agri-food sector in the peri-urban area of Barcelona and Madrid.
\end{abstract}

Keywords: state of alarm; COVID-19; agroecology; informal economy. 


\section{La agroecología en el marco de la crisis del COVID-19}

El 31 de enero de 2020 se dio a conocer en España el primer paciente afectado por coronavirus (COVID-19). El 9 de marzo el Ministerio de Sanidad declaró la contención reforzada y el 13 de marzo el Estado español decretó el estado de alarma. Todas las autoridades civiles de la Administración Pública, así como los cuerpos de seguridad autonómicos o locales quedaron bajo las órdenes del Gobierno. Las vidas de la mayoría de las personas se vieron afectadas en cuanto a libertad de movimiento y circulación. ${ }^{1}$ El Estado, a través de sus aparatos limitó la circulación de vehículos y personas, y restringió las salidas del hogar a ciertas actividades como son la compra de alimentos o de medicamentos. Asimismo, prohibió la celebración de festividades y suspendió los mercados locales, cerró tiendas, bares y restaurantes. El estado de alarma supuso una situación sin precedentes respecto a la paralización de las actividades económicas. Las cadenas de suministro agroalimentarias registraron una gran presión en cuanto a producción y distribución en sus redes regionales y nacionales.

Estos acontecimientos se enmarcan en un contexto de expansión neoliberal, que ha supuesto el cambio de modelo hacia la agricultura y ganadería a gran escala. El modelo agrario desarrollado a partir de los años 60 se ha caracterizado por una fuerte industrialización y por la creciente dependencia de los agroquímicos y los combustibles fósiles (Ploeg, 2010; Wolf, 1982). En este escenario, la industrialización ha incidido en la monetarización de la economía agraria. Después de la crisis financiera de 2008, los organismos de desarrollo y el mundo corporativo han mostrado un renovado interés hacia la agricultura (McMichael, 2012). Además, se ha producido la mecanización del trabajo agrícola que ha provocado una progresiva disminución de la población que se dedica exclusivamente a la agricultura (Homs; Martínez, en prensa), así como también una gradual descampesinización y conversión

1 La concepción y la toma de datos para la elaboración de este artículo ha tenido lugar en los meses de abril y mayo del 2020, durante la denominada "Fase 0" de las medidas impuestas por el estado de alarma. El confinamiento en las casas de la mayoría de la ciudadanía se encontraba vigente y la libertad de movimiento se hallaba restringida. 
del sector agroalimentario hacía otras actividades (Gascón; Milano, 2018). Frente a estas circunstancias, la agroecología se propone como una potencial alternativa frente a la producción agroindustrial neoliberal (Altieri; Nicholls, 2020) y ha sido revindicada como contraparte desde los movimientos sociales. Tal como detallan Guzmán, González de Molina y Sevilla (2000), la perspectiva agroecológica incluye no solo el uso de unas determinadas técnicas agrícolas orientadas a la producción de alimentos, sino que también considera las relaciones socioeconómicas, socioculturales y políticas que se establecen en la agricultura a lo largo del tiempo en diferentes territorios.

A partir de la fragilidad socio-ecológica puesta de manifiesto durante la emergencia sanitaria se ha producido una renovada atención acerca de las potencialidades de la agroecología en torno a su capacidad para prescindir de los pesticidas, revitalizar pequeñas granjas, crear sistemas alternativos de producción y reformar la agricultura urbana (Altieri; Nicholls, 2020). Mucho antes de la pandemia, ya existía una enraizada crítica hacia la agricultura industrial, su dependencia de los insumos no agrícolas y sus grandes riesgos para la salud humana y para el ecosistema (Altieri et al., 2015). En el contexto europeo, las prácticas agroecológicas se han circunscrito en un marco más amplio de política económica y de restructuración de las relaciones de producción de la agricultura actual (Ploeg, 2020). Para este autor, las prácticas agroecológicas han surgido como consecuencia del aumento de la dependencia de los agentes externos, los bancos, las industrias y como respuesta a la desigualdad de las cadenas de suministro agrícola. Finalmente, la necesidad de una transición del régimen alimentario -representada por el paso de la agroindustrialización a la agroecología- basada en el lugar (entendido como lo opuesto a food from nowhere relacionado con la producción agroindustrial) ofrecería "una inversión de tendencia de la dramática huella ecológica y de la acumulación de capital a cualquier precio" (McMichael, 2012, p. 290).

El concepto de agroecología ha sido definido como una disciplina científica, una práctica agrícola y un movimiento. Sus enfoques han variado desde acepciones más ecológicas hasta aproximarse a aspectos sociales y culturales. Algunos elementos que caracterizan la agricultura agroecológica identificados por la FAO son la diversidad, las sinergias, la eficiencia, la resistencia, el reciclaje, la co-creación y el intercambio de conocimientos, los 
valores humanos y sociales, la cultura y tradiciones alimentarias, la gobernanza responsable y la economía circular y solidaria (FAO, 2019). En la actualidad la presencia de las iniciativas autodefinidas como agroecológicas, de pequeña escala en los territorios rurales y periurbanos juega un papel significativo en el aprovisionamiento de alimentos, es decir, abastecen de alimentos a una parte de la población sin priorizar la lógica de la maximización de beneficios por encima del cuidado del medio ambiente (Escribano et al., 2020). Sin embargo, la viabilidad de las iniciativas no es un asunto sencillo debido al contexto político y económico en el que se encuentran insertas. En otras investigaciones ${ }^{2}$ se ha podido comprobar cómo la detección de epidemias en el ganado (lengua azul, gripe aviar...) ha tenido efectos restrictivos sobre prácticas tradicionales ganaderas. Actividades que no se encontraban reguladas por las administraciones públicas, pero sí desde la gestión de los comunes (es decir, instituciones regulatorias formadas por los mismos usuarios), se han visto forzadas a cumplir con nuevas normativas europeas burocratizando las prácticas tradicionales y transformando las propias prácticas, mayoritariamente hacia la economía de mercado.

Estas prácticas tradicionales están relacionadas con lo que puede ser denominado estilo de vida campesino. Desde una visión centrada en la economía, el estilo de vida campesino sería aquel que prioriza en su orientación económica la reproducción de la vida por encima de la participación en la economía de mercado (cf. Narotzky, 2016; Ploeg, 2010). Al hablar de campesinado cabe mencionar la esencialización existente de la figura del campesinado también presente desde la propia agroecología y el movimiento de soberanía alimentaria. La agricultura campesina se puede convertir erróneamente en sinónimo de producción sostenible para el consumo local. En contextos como el altiplano rural de Ecuador, campesinos indígenas han agrietado esta visión romántica evidenciado prácticas de producción que emplean productos químicos y orientadas a la exportación (Soper, 2020).

2 "Rural self-management initiatives and public policies: A conceptualization of neo-peasants". IP: Agata Hummel, investigadora Paula Escribano. Financiado por la Wener-Gren Foundation ID 78071 (2019-2021). 
Esta visión escéptica, se ha fundamentado también en la crítica hacia los principios de la agroecología y de la soberanía alimentaria y su capacidad de que la agricultura de bajos insumos y mano de obra intensiva pueden alimentar la actual población mundial (Bernstein, 2014). Asimismo, se advierte que la agricultura campesina no debe necesariamente mezclarse con la agroecología local (Soper, 2020) y, a la figura de los campesinos, no deben atribuirse per se principios como la cooperación, la reciprocidad o el igualitarismo (Bernstein, 2014).

A nivel político, las prácticas agroecológicas están insertas en un contexto de gubernamentalidad neoliberal que cada vez más implica las 'técnicas del yo', es decir, se apoya en tecnologías y métodos que propician que los individuos se gobiernen y administren a sí mismos a través de diversas prácticas y normas (Shore, 2010). Estas tecnologías van a la par con las políticas del libre mercado las cuales promueven la competitividad y libre circulación de bienes. Sin embargo, la retracción del Estado como agente controlador es aparente. Como veremos a lo largo de este artículo, las lógicas de gobernanza basadas en la regulación de la vida ciudadana a partir de la creación de nuevas normativas o el control policial siguen presentes y se activan en el contexto de la crisis sanitaria. Es lo que Foucault (2003) nombraba el poder disciplinario.

En el escenario de la pandemia generada por el COVID-19, la antropología cuenta con una larga trayectoria sobre el estudio de las crisis y los desastres y ha problematizado cómo los sistemas socioculturales se adaptan y se ajustan a ciertas transformaciones de su entorno natural y social (Oliver-Smith, 1996, 1999). La situación ocurrida a raíz de la declaración del estado de alarma da pie a reflexionar sobre los modelos productivos que son privilegiados para los aparatos del Estado como formas de abastecimiento así como sobre los mecanismos de exclusión/inclusión estatal y de mercado. El presente artículo analiza cómo las pequeñas iniciativas agroecológicas han vivido el cambio en las regulaciones de la vida pública, qué retos han enfrentado y qué efectos han tenido las medidas sobre su actividad principal: la producción y distribución de alimentos a escala local. En especial, la investigación indaga sobre el papel de la economía informal en su doble función de amortiguador y limitante en el aprovisionamiento y propio funcionamiento de las iniciativas agroecológicas. 


\section{Crisis y economía informal}

Como consecuencia de las medidas tomadas para evitar el colapso de la sanidad pública, la crisis sanitaria ha derivado en una crisis social y económica. Los escenarios de crisis no son novedosos en el marco del capitalismo neoliberal que busca constantemente nuevas formas de extracción de plusvalía de los recursos naturales, humanos y de las diferentes dimensiones del trabajo. Las crisis no representan únicamente recesiones económicas sino más bien crisis de estabilidad social (De Angelis, 2013) y alimentan la reproducción del modelo económico y social (Harvey, 2014). Históricamente, las crisis cíclicas incrementan la desigualdad como consecuencia de la concentración y acumulación de riqueza (Piketty, 2014). Además, a medida que el trabajo se ha vuelto más atomizado y flexible, se reduce el llamado estado de bienestar (Bauman, 2015; Valenzuela; Reygadas; Cruces, 2015). En este sentido, resulta relevante indagar sobre el papel que juegan los gobiernos y los sistemas que regulan la vida social a través de normas legislativas causantes de desigualdades y de la precarización de la vida, entendida como la inestabilidad del empleo y de las condiciones de vida en general (Standing, 2014). Por último, indagar acerca de las políticas públicas permite observar los privilegios y la exclusión que se producen entre las diferentes clases sociales y de qué manera.

Los escenarios de crisis requieren reajustes en las relaciones de clase y poder y restructuran los sistemas de gobernanza para dar vida a nuevos espacios de crecimiento y acumulación de capital (De Angelis, 2013). Durante las crisis, los movimientos de personas, bienes, objetos e información y las relaciones intrínsecas de poder, se proponen como emblemáticos espacios de problematización. Además, en el marco de los problemas antropológicos de ensamblajes globales (Collier; Ong, 2005), el articulo problematiza las relaciones de producción y consumo en contextos de informalidad en tiempos de crisis. En los escenarios socioeconómicos de las crisis se potencia el trabajo y la economía informal a pesar de que no siempre sean rentables (Menni, 2004). Desde la antropología, las primeras aproximaciones sobre economía informal comenzaron en los años sesenta (Martínez Veiga, 2004) pero no fue hasta las investigaciones sobre el trabajo urbano informal en Ghana de Hart (1973) que el concepto de sector informal se estableció como campo de investigación. 
Desde las primeras teorizaciones, los caminos del concepto de informalidad desde las "oportunidades informales" hasta el propio concepto de "sector informal" han recorrido un marco histórico relacionado con la marginalidad (Rabossi, 2019). A partir de las primeras perspectivas que tomaban en cuenta las oportunidades de ingresos informales (Hart, 1973), la economía informal ha tenido un gran éxito durante más de tres décadas como un dispositivo conceptual referido a todo lo que ocurre fuera de la burocracia (Hart, 2006). Según el autor Keith Hart, es necesario investigar y entender el funcionamiento de estas formas sociales que surgen más allá de la ley en lo que llamamos economía informal.

Desde entonces ha resultado relevante estudiar la informalidad, como muestran desde los ochenta las crisis en los países latinoamericanos (Biles, 2009). Por ejemplo, en Argentina en 2002 las tasas de trabajo informal y la propia naturaleza y tipología de trabajo aumentaron sin precedentes (Whitson, 2007). En Asia, América Latina y en África subsahariana la creciente vulnerabilidad laboral, la disminución de la demanda y la reducción de los salarios de mujeres trabajadoras informales (que representan la mayor fuerza de trabajo) han protagonizado los tiempos de crisis económicas (Horn, 2010). En algunos contextos, tales como el latinoamericano, desde la década de los ' 90 la existencia de un amplio sector no estructurado e informal abría un largo camino de formalización de la economía y de formalización de una mayor clase asalariada (Lautier, 1990). En las últimas décadas, los debates sobre trabajo informal y su relación con el desarrollo del empleo formal han sido objeto también de investigaciones empíricas y desarrollos teóricos en Europa (Pfau-Effinger; Flaquer; Jensen, 2010).

De manera general, la noción de trabajo informal hace referencia a cuestiones relacionadas con lo legal, la fiscalidad, las licencias y las autorizaciones. Se hace referencia a la economía formal como aquella en la que se realiza un trabajo asalariado, mientras que la economía informal es aquella basada en el autoempleo y sin una mano de obra permanente y fija que reciba recompensas (Hart, 1973, p. 68). El llamado sector informal ha sido incorporado en un ámbito más amplio como es el de economía subterránea, que englobaría la economía ilegal (que incluye las prácticas prohibidas por la ley), la economía no declarada (que se refiere a la dimensión fiscal), la economía no registrada (que incluye las actividades no registradas en las 
instituciones gubernamentales) y la propia economía informal, definida como "actividades económicas que eluden los costos y están excluidas de los beneficios y los derechos incorporados en las leyes y normas administrativas relativas a la propiedad, licencias comerciales, contratos de trabajo, agravios, crédito financiero y sistema social de seguridad" (Feige, 1990, p. 992). En este marco, las actividades informales cubren un largo abanico, desde operaciones marginales hasta grandes empresas. Sin embargo, no existe homogeneidad entre las prácticas no legalizadas, haciendo difícil la comparativa solo por su carácter fuera de la normativa. Las actividades fuera de la ley han sido clasificadas como legítimas e ilegítimas, siendo estas últimas las sancionadas no solo por la ley, sino también moralmente (Hart, 1973). En este contexto, "el sistema de los valores burgueses que toma forma en los códigos de leyes de los países puede no ser coincidente con el concepto de legitimidad prevaleciente en algunas subculturas de la sociedad" (Hart, 1973, p. 74).

En el caso de las actividades agroalimentarias, la economía informal está relacionada directamente con la actividad económica no dada de alta ni registrada en las instituciones del Estado. Dentro de las actividades no registradas podemos encontrar transacciones de mercado y transacciones fuera del mercado. En estas últimas, entraría la economía de autoabastecimiento. La actividad económica fuera de los mercados supone entre un 30 y un $50 \%$ de la actividad económica incluso en las sociedades Occidentales y puede ser parte de las "economías diversas", proyectos de autonomía económica y de experimentación con una orientación anticapitalista (Gibson-Graham, 2008). Por todo ello, analizar la función de la economía informal de las pequeñas iniciativas agroecológicas mejora la comprensión de las reestructuraciones y desequilibrios experimentados por el sector a escala local.

\section{Metodología}

Para la elaboración de este artículo se ha utilizado una metodología cualitativa. Se han analizado nueve casos de estudio insertos en un contexto en el que ya se había realizado trabajo de campo etnográfico previamente entre 2013 y 2019, con especial intensidad en el caso de Cataluña (Escribano et al., 2020). El interés se ha centrado sobre casos de pequeños productores, tanto 
agricultores como ganaderos que incorporaran una visión agroecológica en la iniciativa y se hallaran en la zona periurbana de Barcelona o de Madrid. Se han analizado cuatro casos en Barcelona y cinco casos en Madrid. La elección de estas dos ciudades se fundamenta por ser los centros urbanos con mayor número de habitantes e iniciativas agroecológicas en el Estado español. La recolección de datos ha sido a través de entrevistas semiestructuradas complementadas con la participación en dos grupos de WhatsApp y el análisis de materiales secundarios - tales como comunicados y videos realizados por los y las informantes.

El primer grupo de WhatsApp se localiza geográficamente en Cataluña y cuenta con 170 participantes. El grupo está orientado a la temática de la agricultura y ganadería campesina. El segundo grupo se localiza entre Cataluña, Aragón y el País Vasco, cuenta con 73 participantes y se orienta a temas de ganadería extensiva. ${ }^{3}$ Los grupos de WhatsApp se formaron con el objetivo de formar redes locales e intercambiar noticias de interés colectivo. Además, sirven como comunidad online de apoyo mutuo para resolver dudas y compartir ideas e informaciones.

Las entrevistas semiestructuradas se han realizado de forma online, presencial y telefónica. En total se han realizado 11 entrevistas, nueve a las personas responsables de las iniciativas y dos a actores clave del sector: una empresa distribuidora de producto alimenticio ecológico en Cataluña y una cooperativa centrada en la dinamización de iniciativas agroecológicas en Cataluña (Véase Tabla 1). Las entrevistas han sido grabadas durante los meses de abril y mayo. Con la finalidad de proteger el anonimato de las personas participantes en esta investigación, tanto los nombres como las localidades que se presentan a lo largo del trabajo han sido sustituidos por pseudónimos. 


\begin{tabular}{|c|c|c|c|c|c|c|c|c|c|}
\hline 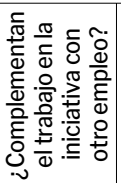 & 우 & z & $\bar{\omega}$ & $\bar{\omega}$ & z & is & $\bar{\omega}$ & $\bar{n}$ & 으 \\
\hline 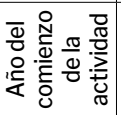 & ָี & ్ㅗ & $\stackrel{m}{\stackrel{\sim}{\sim}}$ & శ్ & $\stackrel{\text { ㅇ }}{\stackrel{\circ}{\sim}}$ & $\stackrel{m}{\stackrel{\sim}{\sim}}$ & $\stackrel{m}{\stackrel{m}{\sim}}$ & 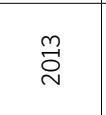 & ষ্ণ \\
\hline 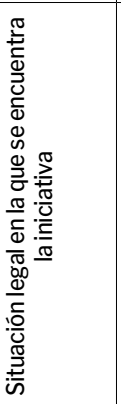 & 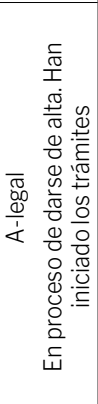 & 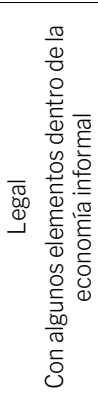 & 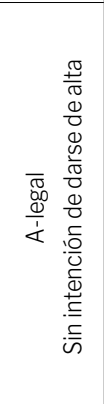 & 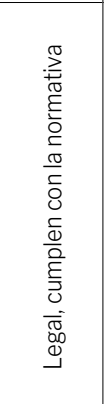 & 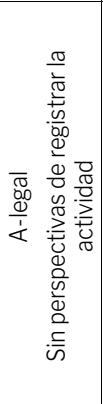 & 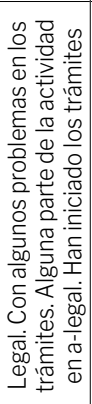 & 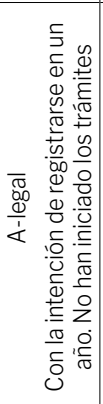 & 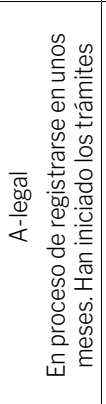 & 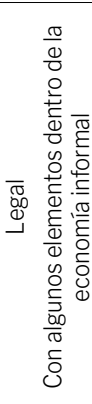 \\
\hline 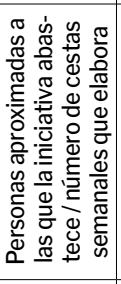 & 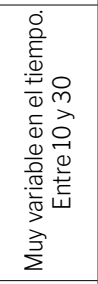 & 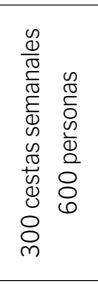 & 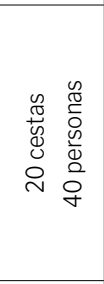 & 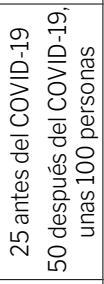 & 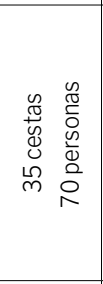 & 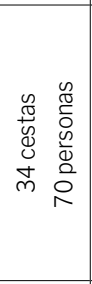 & 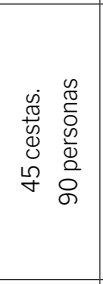 & 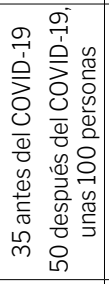 & 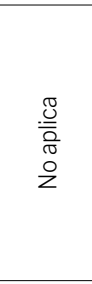 \\
\hline 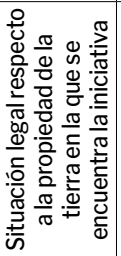 & 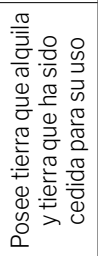 & 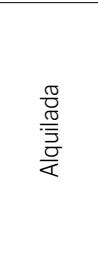 & $\begin{array}{l}\frac{\pi}{0} \\
\frac{\pi}{7} \\
\frac{\bar{U}}{0}\end{array}$ & 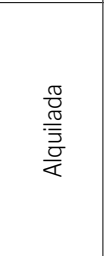 & 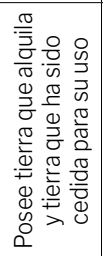 & 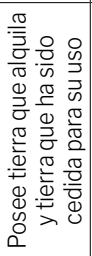 & 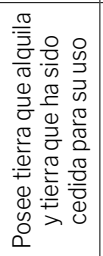 & $\begin{array}{l}\frac{0}{0} \\
\frac{0}{0} \\
\frac{\bar{z}}{\frac{\pi}{\alpha}}\end{array}$ & 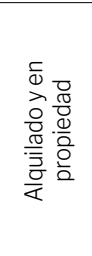 \\
\hline 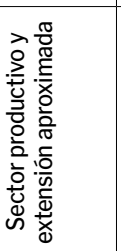 & 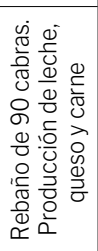 & 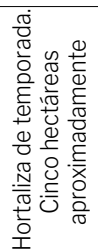 & 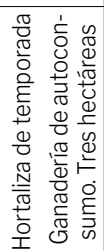 & 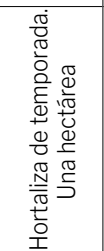 & 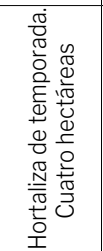 & 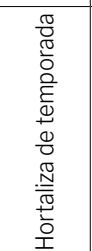 & 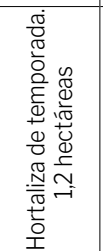 & 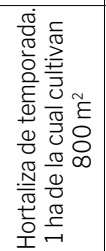 & 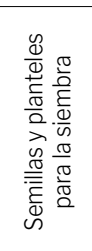 \\
\hline 离 & $\begin{array}{l}\frac{\pi}{5} \\
\frac{\sqrt{5}}{\sqrt[T]{0}} \\
\frac{\pi}{\pi} \\
0\end{array}$ & $\begin{array}{l}\frac{\pi}{5} \\
\frac{\sqrt{5}}{\sqrt[T]{\pi}} \\
\frac{\pi}{0} \\
0\end{array}$ & $\begin{array}{l}\frac{\pi}{5} \\
\frac{\sqrt{5}}{\sqrt[T]{0}} \\
\frac{\pi}{\pi} \\
0\end{array}$ & $\begin{array}{l}\frac{\pi}{5} \\
\frac{\sqrt{5}}{\pi} \\
\frac{\pi}{\pi} \\
U\end{array}$ & $\begin{array}{l}\frac{0}{\frac{0}{\bar{l}}} \\
\frac{\pi}{2}\end{array}$ & $\begin{array}{l}\frac{\overline{0}}{\overline{\frac{V}{7}}} \\
\frac{\pi}{2}\end{array}$ & $\begin{array}{l}\frac{\overline{0}}{\overline{\frac{V}{2}}} \\
\frac{\pi}{2}\end{array}$ & $\begin{array}{l}\frac{\overline{0}}{\overline{2}} \\
\frac{\pi}{2}\end{array}$ & $\begin{array}{l}\frac{\overline{0}}{\overline{\frac{1}{0}}} \\
\frac{\bar{\pi}}{2}\end{array}$ \\
\hline $\begin{array}{l}\text { on } \\
\text { ర్ }\end{array}$ & $\frac{\frac{\sqrt{0}}{\sqrt[N]{\pi}}}{\sum}$ & 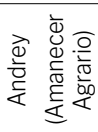 & $\begin{array}{l}\stackrel{ }{\bar{ர}} \\
\stackrel{0}{ㅇ}\end{array}$ & 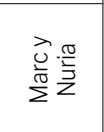 & 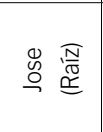 & 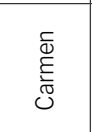 & $\begin{array}{l}\overline{\bar{Q}} \\
\stackrel{0.00}{\Sigma}\end{array}$ & : & 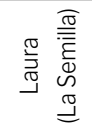 \\
\hline
\end{tabular}




\section{Las incidencias de las iniciativas agroecológicas en los entornos periurbanos de Madrid y Barcelona}

Como punto de partida, cabe señalar que la mayoría de las iniciativas agroecológicas estudiadas no tienen el terreno en propiedad. Las tierras son alquiladas, cedidas u ocupadas, a veces combinando diferentes modelos. En siete de las nueve iniciativas, ganadería y agricultura se complementan, si bien no siempre ambas son utilizadas para la comercialización. Las iniciativas agroecológicas observadas tienden a combinar prácticas de agricultura con prácticas de ganadería y se orientan económicamente a lo que ha sido nombrado como lógica campesina buscando la subsistencia por encima de la especialización y la búsqueda del máximo beneficio en la economía de mercado. En estas últimas la figura principal es el agricultor profesional. Las iniciativas agroecológicas que cultivan hortaliza o plantel para su venta $(n=8)$ utilizan entre una y cinco hectáreas de cultivo. La iniciativa ganadera $(n=1)$ que comercializa el producto, cuenta con 90 cabezas de ganado. De forma general, las nueve iniciativas estudiadas abastecen de producto alrededor de 50 hogares, aproximadamente 100 personas. En el caso de Amanecer Agrario, una iniciativa del periurbano de Barcelona, la cifra llega hasta las 600 personas. Siete de las nueve iniciativas comenzaron su actividad comercial en torno a 2012, en medio de un escenario de crisis económica en España, aunque muchas de las personas habían estado implicadas en iniciativas similares con anterioridad. Respecto a las jornadas y a los ingresos, tan solo Andrey de la cooperativa Amanecer Agrario trabaja a jornada completa y supera los $1000 €$ de salario. El resto trabajan a media jornada y jornada completa cobrando entre $400 \mathrm{y}$ $700 €$ al mes. En la mayoría de estos casos necesitan complementar su trabajo en la iniciativa con otras fuentes de ingresos.

El modelo de distribución del producto es la "cesta", una caja de verduras que bien puede ser "cerrada" es decir, el consumidor no escoge el contenido o "abierta", se pueden seleccionar los productos. La distribución se nombra coloquialmente como "el reparto". El tipo de consumidores varía entre (1) grupos de consumo, agrupaciones de personas que se auto-organizan para acceder a un producto agroecológico, (2) “cooperativas de consumo”, un formato similar pero dado de alta y con estatutos legales. Un ejemplo son los supermercados cooperativos. En este modelo los clientes son socios de las cooperativas (3) Personas a 
título individual. No se organizan en colectivo, pero pueden acudir a un mismo punto para recoger su "cesta".

Respecto a la normativa legal, tres casos están registrados como iniciativas legales y cinco no lo están de los cuales tres desearían tramitar el alta o están en proceso de hacerlo. La informalidad económica en ocasiones es denominada como "a-legal", es decir, no incumplen la norma sino que su actividad se desarrolla dentro de una lógica diferente. Carmen, sobre los 39 años, trabaja junto con su compañero como hortelana en el periurbano de Madrid y definía así lo a-legal:

Pues que no somos nada. Es como que estamos en un limbo, que no somos algo ilegal, somos un grupo de gente que se organiza para tener una huerta y ya está, pero no somos legales. Aunque tú digas que pones un trabajo al mes, al final hay un trabajo que es remunerado y no aparece en ningún lado.

Entre las iniciativas hay diferentes grados de uso de la economía informal. En un extremo están los proyectos que operan mayoritariamente dentro de la lógica informal, esto es, sin poseer una figura legal, no declaran su actividad ni cumplen con la normativa asociada al tipo de actividad que desarrollan. En el otro extremo están las iniciativas que poseen una organización que cumple con la legislación, están registradas y pasan las inspecciones correspondientes, pero utilizan la economía informal para superar ciertos obstáculos económicos o avanzar con la actividad mientras llega, por ejemplo, un permiso. Las motivaciones para no registrar la actividad o realizar algunas prácticas fuera de lo legal van desde el rechazo ideológico en la forma propuesta por las administraciones públicas sobre el manejo de la iniciativa o explotación, hasta la incapacidad de cumplir la normativa por los costes monetarios que esto supone. Miguel, agricultor sobre los 30 años de Madrid, comentaba lo siguiente:

$\mathrm{Si}$, lo estamos mirando, pero es que claro, como manejamos tan poco dinero, digamos que no hay una...si hubiera una cuota de autónomos por ingresos pues podríamos planteárnoslo, pero es que no da. Porque tienes seis meses que pagas $50 €$, pero es que luego son 300 y pico por trabajador, o 200 y pico. Ganando lo que ganamos se te va la mitad del salario. 
Entre las razones por la que le gustaría legalizar la iniciativa Miguel identifica:

Bueno, en esta pandemia no ha pasado nada, pero al menos te podrías mover de forma legal si vuelve a pasar esto y bueno, no estar siempre pendiente de si te pillan, las multas ... que nunca nos ha pasado ¿eh? Pero bueno. Y luego también piensas en la jubilación un poco, en cotizar...

En este escenario, parece que para Miguel lo a-legal no sería una opción ideológica.

Bueno, ideológicamente también puede tener sentido estar dentro del sistema de seguridad social y aportar para que otros también puedan. En todo este tiempo me hubiera gustado estar dado de alta. Pero bueno, si no se puede no se puede, tampoco quiero matarme a trabajar, o trabajar mucho más para esto. Me doy [de alta] si me parece justo lo que pago, si no, no.

Con la entrada del estado de alarma el carácter formal o informal de las actividades ha sido fundamental a la hora de exponer a las iniciativas a una mayor o menor vulnerabilidad y precariedad. En este contexto, la economía formal ha sido amparada por las medidas extraordinarias, mientras la informal ha sido invisibilizada y excluida como estrategia para el abastecimiento, pese a ser una realidad que permite autoemplearse y distribuir alimentos a una parte de la población. A continuación, se destacan los principales cambios y retos observados en las iniciativas.

\section{El efecto del COVID-19 en los proyectos agroecológicos}

Durante el estado de alarma ha existido un aumento en la demanda de "cestas ecológicas" tanto en Madrid como en Barcelona. Los proyectos dados de alta -bien como autónomos agrarios o trabajando para terceros- que estaban en disposición de responder al aumento de la demanda con otros productos ajenos a su huerta han notado un considerable crecimiento de sus ventas. Sin embargo, las iniciativas que venden su producto en grupos de consumo y que planifican sus cultivos por temporadas apenas han notado la variación. 
Carmen comentaba: “Algún vecino nos ha preguntado: Oye, esto está muy fresco ¿de dónde viene?, ¿cómo te puedo comprar? Y yo le he dicho: No, esto no es exactamente comprar, esto es otra cosa". Lo que Carmen hace no es vender, sino abastecer de hortalizas a un colectivo. Según comenta, las relaciones que se establecen entre las personas no son las mismas ni tampoco su nivel de implicación. En el caso de Carmen las personas que reciben las verduras tienen obligaciones en el huerto en el que Carmen trabaja, y pueden tomar decisiones sobre el desarrollo y funcionamiento del mismo. Además, el colectivo de Carmen no compra verduras para "completar las cestas". Si un mes tienen menos productos, el grupo de consumo asume la falta. Se organizan por cuotas fijas calculadas en función de los gastos que tiene el colectivo, donde incluyen "las asignaciones de las hortelanas", el dinero que reciben a final de mes. El precio del producto en el mercado no es importante para marcar el precio de la cuota.

Entre los casos que doblaron o triplicaron su venta está el caso de Marc y Nuria, del periurbano de Barcelona. Tienen un proyecto dado de alta que lleva ocho años combinando el reparto de cestas "puerta a puerta" con vender en una parada los sábados: "Ahora no hay mercado, pero hemos doblado pedidos, como 50 pedidos semanales". Antes del estado de alarma tenían entre 20 y 25 pedidos semanales. Facturaban entre 500 y $600 €$ a la semana y con esto no podían cubrir ni un sueldo. Durante el estado de alarma han facturado entre 900 y $1000 €$. Ahora les da para cubrir un sueldo y algo más. Otro caso es el de Amanecer Agrario, una iniciativa también dada de alta de producción y distribución de fruta y verdura ecológica que comenzó en 2012 cerca de Barcelona. En su caso no ha aumentado el número de compradores sino la cantidad de producto que piden en cada cesta. Han podido hacer frente a la demanda porque trabajan en red. El proyecto está asociado con otras huertas de ideología similar a la suya y pueden abastecerse de más hortalizas si lo necesitan. El aumento de la demanda aparece justificado por tres factores: (1) el cuidado de la salud y el cuerpo, de especial importancia frente al riesgo que representa la enfermedad. (2) El trato directo con los clientes y las redes sociales informales y (3) la comercialización puerta a puerta. Este último factor ha sido clave para la adaptación de las iniciativas a la nueva normativa, aportando seguridad y comodidad a los consumidores. Según Alba, socia fundadora de La Despensa y dedicada desde hace más de 20 años a la distribución de fruta y verdura ecológica en Cataluña, los que denomina como "nuevos 
rurales" han sido muy rápidos en cambiar el chip y adaptarse a las necesidades del mercado:

Los de ecológico han sido muy rápidos, han tenido un "clic" de venga ¿no puedo venderlo por ese canal? pues venga, hago lo que sea. Se han dedicado a vender al consumidor final. Para el consumidor final es bueno, te lo llevan a casa. Conozco bastante gente que hace cestas, porque hacían mercados. Ha sido su salida.

David es el ejemplo de lo que Alba nombra rapidez de adaptación. En su proyecto han pasado de vender 35 cestas semanales a 50. No tienen "papeles", pero están pensando en tramitarlos. Según comenta David, la primera semana todas las ventas quedaron paradas porque los grupos de consumo tenían los locales cerrados. "Entonces decidimos empezar con el reparto a domicilio y ha sido la explosión”. David, también trabaja en red como Amanecer Agrario.

\section{El doble papel de la economía informal en tiempos de crisis: amortiguador y limitante}

En general se ha observado cómo las prácticas económicas informales de las iniciativas agroecológicas estudiadas han actuado en dos sentidos. Por un lado, han tenido un efecto amortiguador, es decir, han ayudado a recuperar el equilibrio después de primeras semanas de caos durante el estado de alarma y han permitido hacer frente a la creciente demanda de producción. Esto ha ocurrido principalmente en los proyectos que tienen la mayor parte de su producción dentro de la economía formal. Por otro lado, la economía informal ha actuado como limitante, es decir, ha hecho que las iniciativas "sin papeles" hayan tenido que modificar la distribución hasta en algunos casos suprimirla, cambiar los horarios de reparto, crear coartadas y sufrir miedo e incertidumbre a la hora de realizar su actividad de abastecimiento.

En su papel amortiguador, ciertas prácticas informales han permitido la continuidad de actividades tales como "el reparto". Durante el estado de alarma la mayoría de las cooperativas y grupos de consumo cesaron su actividad. En consecuencia, la mayoría de las iniciativas tuvieron que realizar la distribución del producto en la calle, exponiéndose a sanciones y comentarios de los 
vecinos. De esta forma asumieron la obligación de buscar soluciones y límites a las problemáticas que se iban desarrollando, acorde a la idea de autogobierno y autoadministración presente en las prácticas de gubernamentabilidad neoliberal, como se mencionaba al inicio del artículo. Andrey comentaba al respecto:

Yo llego, abro la furgoneta, la gente me espera o yo les espero a una hora y la gente viene. No es legal que vayan a buscar la verdura. Lo hacemos pim-pam, es como un mercadillo así... creo que no es legal, pero de otro lado ¿cómo salir de esta situación? no podemos dejar a la gente que llevan 10 años consumiendo, conocen el proyecto, tienen confianza... No teníamos otra opción.

Otra forma de afrontar las problemáticas ha sido la prestación de ayuda. Los proyectos con menos probabilidades de ser sancionados han ayudado a los proyectos más expuestos. La ayuda principalmente ha consistido en la distribución del producto a través de la venta por canales informales. Los vecinos de Marc y Nuria, por ejemplo, tenían un rebaño de cabras y producían quesos. A raíz de las medidas impuestas por el estado de alarma "estaban vendiendo menos de la mitad que vendían normalmente". Marc y Nuria decidieron ayudarles añadiendo los quesos a las cestas de verduras pese a no cumplir con la normativa por no tener una nevera especial. También David ha sumado a su reparto queso fresco y pan: "A la gente de los quesos lo que les ha pasado es que no están dados de alta ni nada y tenían miedo de venir a Madrid. Entonces una vez al mes me dan los quesos y los huevos, los monto directamente y los reparto".

Por otro lado, el encontrarse "fuera de la ley" o en una situación legalmente no muy clara ha tenido consecuencias desastrosas para la continuación de ciertas actividades. Ha sido el caso de los ganaderos Fede, Maria y Jose. Desde hace un par de años intentan legalizarse. La zona periurbana de Barcelona es un lugar difícil en términos de legalización de un rebaño. La cercanía de la ciudad provoca muchas restricciones administrativas difíciles de cumplir. Mientras realizaban los trámites vendían leche y carne informalmente para tener "un mínimo de ingresos y hacer viable el proyecto". Con la entrada del estado de alarma la gente no podía salir de casa a por leche o queso y ellos tampoco podían realizar el transporte porque no tenían permiso para ello. Como consecuencia continuaron trabajando con el rebaño sin ingresos. Frente 
esta situación Jose se vio obligado a abandonar el proyecto. María tuvo que mudarse a vivir en una tienda de campaña cerca del rebaño para poder seguir trabajando. No disponía de ningún comprobante que autorizase su movilidad durante el confinamiento.

Las dificultades ejemplificadas para comercializar el producto de ganadería agroecológica durante el estado de alarma también se reflejaron en los grupos de WhatsApp. Una parte de la producción cárnica encontraba salida en restaurantes o eventos sociales. Durante las primeras semanas de regulación algunas explotaciones tiraron la leche que no vendían. El pequeño sector ganadero no se ha mostrado tan flexible como el de la verdura agroecológica ante la quiebra de sus canales de distribución, debido a la sobre-regulación en la que está envuelto el sector.

Para Joan, la venta de verduras es un complemento a su economía. Trabaja como agricultor para la comercialización y ganadero para el autoconsumo y vive en una masía a unos 25 kilómetros de Barcelona. No tiene su actividad registrada. Joan paró la distribución de verduras durante el estado de alarma por razones de salud y de seguridad, pero necesitaba comprar grandes cantidades de plantel para preparar la siguiente temporada. Joan relata su sensación frente a los controles.

Es muy arbitrario, muy incierto, es depende de a quién te encuentres, de lo que lleves. No hay un documento, no está claro. Y también es parte de lo que quieren hacer. Esa arbitrariedad, esa inseguridad de si estás cometiendo un delito o no... es el poder absoluto del policía. Me ha dicho un amigo que lleva pan, que cuando te ven con comida entienden que trabajas en la alimentación [y no te multan].

El ejemplo de Joan muestra cómo el miedo y la incertidumbre ante las sanciones han actuado también como limitantes de la actividad comercial y de abastecimiento. Frente a este miedo las iniciativas han reaccionado de diferentes formas. En el caso de Miguel y su socio, en la periferia de Madrid, la actuación fue esperar a que les pusieran la primera sanción y después valorar la situación. Su iniciativa es a-legal y cuentan con una "caja de resistencia" es decir, un fondo especial para hacer frente a los imprevistos. David, que también continuó con su actividad cuenta su vivencia: "He perdido tres meses de vida, ya te digo, cuando iba por las calles y me parecía ver una luz azul, el corazón se me 
salía. Era como un jahora sí, ahora ya sí!". David además comenta que al principio no tenía coartada preparada. Luego consiguió un papel que le habilitaba como productor oficial, gracias a una amiga que le hizo un justificante como si trabajara para ella.

Otro ejemplo es el de la asociación La Semilla en Madrid que se dedica a rescatar la biodiversidad agrícola. La asociación es legal, pero desarrolla un área de trabajo en la a-legalidad con las semillas y el plantel. No ha conseguido el título de obtentor y multiplicador de material vegetal porque la Comunidad de Madrid le exige como requisitos tener unas instalaciones y maquinaria como si fuera una empresa grande de semillas. Sin embargo, es un proyecto artesanal pequeño. La Semilla también comercializa sus productos de forma a-legal porque produce variedades tradicionales, que no están registradas. La persona que repartía el plantel con la furgoneta disponía de un documento que certificaba su trabajo legal en la asociación pero el reparto del plantel, al ser a-legal, no se encontraba entre sus responsabilidades.

Él tenía un papel que decía que estaba contratado por la asociación. Pero este papel lo hicimos para que pudieran moverse al invernadero o a otras tareas agrícolas. Pero claro, para repartir es otra cosa. Intentaba no sacarlo [el papel], intentaba no utilizarlo. A veces le paraban y él decía que iba a hacer otras cosas, pero no utilizaba su verdadero trabajo y lo que estaba haciendo por si acaso investigaban más (Sandra).

La producción de semillas y plantas en viveros es una actividad más controlada que la producción de verdura, según nos relató Sandra y nos sugiere el análisis de las diferencias en el marco legal de la producción de verdura, semilla y plantel (España, 2006). El ejemplo del concepto de la trazabilidad alimentaria aplicado en principio a los productos de origen animal o genéticamente modificados nos indica que la producción de verdura hasta hace poco estaba exenta al control más estricto en cuanto al proceso de producción (Agencia Española de Seguridad Alimentaria y Nutrición, 2009). En actualidad se intenta aplicar las normas de trazabilidad alimentaria también a los productos vegetales, pero como nos indica uno de los trabajadores del Departament d'Agricultura, Ramaderia, Pesca i Alimentació (DARP) de Cataluña, de momento no se aplica sanciones por su ausencia (entrevista con el empleado del DARP 22.09.2018). Por 
esa razón la comercialización del plantel ha sido una actividad más delicada durante el estado de alarma. El estrés que suponía repartir el plantel ha tenido un impacto negativo en la salud de la persona responsable de este trabajo.

El miedo y la incertidumbre también han jugado un papel decisivo en el caso de los colectivos de autoabastecimiento, como es la Raíz en Madrid. El no reconocimiento por ley de su actividad de autoproducción ha actuado como limitante para el abastecimiento de hortaliza de temporada funcionando como una forma de poder disciplinario en el sentido foucaultiano. José comentaba al respecto:

No sabemos si podemos o no seguir con la actividad, pero nosotros hemos seguido funcionando. En las diferentes "pantallas" que hemos vivido del confinamiento nosotros hemos considerado que lo que estábamos haciendo estaba permitido. Simplemente producimos comida. Independientemente del estatus legal o de las restricciones, pensamos que nuestra actividad recogía el espíritu de las cosas que estaban permitidas. Esto es tan marciano que por mucho que intentes hacer las cosas de la forma más normal posible no lo es. Vamos a la cosecha y lo intentamos hacer todo rápido, todos separados para no llamar la atención, porque sabemos que la huerta se ve desde la carretera. A pesar de que no hemos tenido problemas no quieres provocar una situación... yo que sé, nunca nos han visto en la cosecha. Las cestas las hacemos escondidos en unos árboles, para no llamar la atención e intentar no provocar esa situación y además con cierta tensión.

Por último, la llegada de las medidas impuestas por el estado de alarma también ha causado cambios en la forma de distribuir la verdura. Si en una situación anterior a el COVID-19 la mayoría de las cooperativas y grupos de consumo recibían la verdura a granel, con la llegada de la emergencia la prohibición de la concentración de gente ha transferido a los productores la carga de dividir los productos en "cestas" individuales. Además, el reparto ha pasado a ser "a domicilio" o en grupos reducidos. Estos cambios han supuesto el aumento de la carga de trabajo. Una estrategia común para afrontar el cambio ha sido la flexibilización, es decir, variar tanto las horas de trabajo como las tareas necesarias para sacar la producción adelante: las mismas personas realizando más trabajo. La autoexplotación, característica de los campesinos, ha 
sido entendida como una condición que permite la mejor adaptación ante variaciones del mercado, además de dotar a las iniciativas de un cierto nivel de independencia (Harris, 2005). Asimismo, esta flexibilización aparece como otra implicación de las crisis cíclicas del sistema económico y su consiguiente aumento de las desigualdades y precarización del trabajo campesino. Como otra cara de la moneda, la autoexplotación puede actuar como ventaja competitiva. En el caso de los proyectos dados de alta y que han realizado la venta dentro de la economía de mercado les ha permitido hacer frente al aumento de la demanda y mantener su posición como iniciativa de abastecimiento. Salvo en algunos casos, las horas extra de trabajo no han sido remuneradas.

\section{Reflexiones finales}

La presente investigación ha mostrado la vivencia de nueve iniciativas agroecológicas del periurbano de Barcelona y Madrid a partir de la declaración del estado de alarma por el COVID-19. La observación ha revelado el papel fundamental de la economía informal en la continuidad de su actividad, como suele ser frecuente en escenarios y contextos socioeconómicos de crisis (Menni, 2004). La investigación ha demostrado que en los casos de estudio los modelos productivos agroecológicos alejados de la economía de mercado no han sido favorecidos por los aparatos del Estado como formas de abastecimiento de la sociedad. Las iniciativas no reguladas en el marco legislativo estatal han encontrado dificultades en la continuación de su actividad. De forma explícita el Estado ha restringido la movilidad de personas impidiendo la circulación de los productos producidos. Por lo tanto, la viabilidad de su actividad se ha visto amenazada. Sin embargo, la restricción no ha sido siempre evidente. Los proyectos agroecológicos han enfrentado inconvenientes tales como el miedo y la incertidumbre, que han actuado como limitantes en sus modelos de distribución.

En este marco, las iniciativas han continuado con su actividad gracias a la implementación de estrategias como la autoexplotación que ha posicionado a las iniciativas en escenarios de vulnerabilidad al continuar con la actividad pese a no tener ingresos. Asimismo, la autoexplotación se ha revelado como estrategia aplicada por las iniciativas con el fin de mantener su crecimiento 
en el momento de la crisis. Igualmente, la manifiesta precarización del trabajo ha resultado de importancia para la sostenibilidad económica de dichos proyectos agroecológicos.

Ambos contextos geográficos muestran la relevancia de estudiar el escenario social y político que crea la intersección entre el Estado y el mercado tanto en la cobertura de las necesidades básicas para la subsistencia de los hogares como en la continuidad de las iniciativas agroecológicas de forma digna (cf. Narotzky, 2016). En concreto, este artículo pone de manifiesto cómo las prácticas agroecológicas se encuentran limitadas a la hora de desempeñar su labor de abastecimiento durante las crisis en el marco de la legalidad estatal. Por un lado, se ha observado como en algunos casos en el momento de la crisis las iniciativas informales han sido excluidas debido al endurecimiento de las restricciones legales. Por otro lado, la informalidad ha jugado un papel amortiguador.

Estas conclusiones se suman al debate de la importancia del papel de la economía informal en el abastecimiento de la sociedad, no solo en los contextos de los países del Sur, sino también en el de las economías y políticas neoliberales del Norte. Asimismo, estos espacios de problematización social y aproximaciones antropológicas resultan de suma importancia para indagar acerca de las complejas relaciones entre movilidad de personas y bienes y el proprio crecimiento de la economía campesina. Por ello, observar y problematizar los ajustes y retos enfrentados por la pequeña agro-producción y las iniciativas agroecológicas, permite comprender en profundidad los antecedentes y las consecuencias de la crisis del COVID-19 así como también cuestionar el propio papel y futuro de las iniciativas agroecológicas dentro de la economía de mercado contemporánea.

\section{Referencias}

AGENCIA ESPAÑOLA DE SEGURIDAD ALIMENTARIA Y NUTRICIÓN. Guía para la aplicación del sistema de trazabilidad en la empresa agroalimentaria. Madrid, 2009. Disponible en: http://www.aecosan.msssi.gob.es/AECOSAN/docs/documentos/ publicaciones/seguridad_alimentaria/guia_trazabilidad.pdf. Acceso: 30 jul. 2020.

ALTIERI, M. A.; NICHOLLS, C. I. Agroecology and the reconstruction of a post-COVID-19 agriculture. The Journal of Peasant Studies, v. 47, n. 5, p. 881-898, 2020. 
ALTIERI, M. A. et al. Agroecology and the design of climate change-resilient farming systems. Agronomy for Sustainable Development, n. 35, p. 869-890, 2015.

BAUMAN, Z. Modernidad líquida. México: Fondo de Cultura Económica, 2015.

BERNSTEIN, H. Food sovereignty via the 'peasant way': a sceptical view. The Journal of Peasant Studies, v. 41, n. 6, p. 1031-1063, 2014.

BILES, J. J. Informal work in Latin America: competing perspectives and recent debates. Geography Compass, v. 3, n. 1, p. 214-236, 2009.

COLLIER, S. J.; ONG, A. (ed.). Global assemblages: technology, politics, and ethics as anthropological problems. Malden: Blackwell, 2005.

DE ANGELIS, M. Does capital need a commons fix?. Ephemera, v. 13, n. 3, p. 603-615, 2013.

ESCRIBANO, P. et al. "Él es emprendedor, pero yo no; yo soy autónomo": autorrepresentación y subsistencia de los neocampesinos en Cataluña. Revista de Antropología Iberoamericana, v. 15, n. 1, p. 129-156, 2020.

ESPAÑA. Ley 30/2006, de 26 de julio, de semillas y plan-tas de vivero y de recursos fitogenéticos. Boletín Oficial del Estado, Madrid, n. 178, p. 28165-28178, 26 jul. 2006.

FAO. The state of the world's biodiversity for food and agriculture. Rome: FAO Commission on Genetic Resources for Food and Agriculture Assessments, 2019. Disponible: http://www.fao.org/3/CA3129EN/CA3129EN.pdf. Acceso: 30 jul. 2020.

FEIGE, E. L. Defining and estimating underground and informal economies: the new institutional economics approach. World Development, v. 18, n. 7, p. 989-1002, 1990.

FOUCAULT, M. “Society must be defended”: lectures at the Collège de France 1975-1976. New York: Picador, 2003.

GASCÓN, J.; MILANO, C. Tourism, real estate development and depeasantisation in Latin America. European Review of Latin American and Caribbean Studies/Revista Europea de Estudios Latinoamericanos y del Caribe, v. 105, p. 21-38, 2018.

GIBSON-GRAHAM, J. K. Diverse economies: performative practices for "other worlds". Progress in Human Geography, v. 32, p. 613-632, 2008.

GUZMÁN, G.; GONZÁLEZ DE MOLINA, M.; SEVILLA, E. Introducción a la agroecología como desarrollo rural sostenible. Madrid: Mundi-Prensa, 2000. 
HARRIS, M. Peasants. In: CARRIER, J. G. (ed.). A handbook of economic anthropology. Cheltenham: Edward Elgar, 2005. p. 423-436.

HART, K. Informal income urban Ghana opportunities and urban employment in Ghana. The Journal of Modern African Studies, v. 11, n. 1, p. 61-89, 1973.

HART, K. Bureaucratic form and the informal economy. In: GUHA-KHASNOBIS, B.; KANBUR, R.; OSTROM, E. (ed.). Linking the formal and informal economy: concepts and policies. Oxford: Oxford University Press, 2006. p. 1-18.

HARVEY, D. Diecisiete contradicciones y el fin del capitalismo. Quito: IAEN, 2014.

HOMS, P.; MARTÍNEZ, B. Dignity and just prices. The moral economies of farming in the age of agro-industry. Disparidades: Revista de Antropología. En prensa.

HORN, Z. E. The effects of the global economic crisis on women in the informal economy: research findings from WIEGO and the Inclusive Cities partners. Gender \& Development, v. 18, n. 2, p. 263-276, 2010.

LAUTIER, B. Wage relationship, informal sector and employment policy in South America. The Journal of Development Studies, v. 26, n. 2, p. 278-298, 1990.

MARTÍNEZ VEIGA, U. Trabajadores invisibles: precariedad, rotación y pobreza de la inmigración en España. Madrid: Catarata, 2004.

MCMICHAEL, P. Food regime crisis and revaluing the agrarian question. Research in Rural Sociology and Development, v. 18, p. 99-122, 2012.

MENNI, A. M. Cómo rebuscársela: trabajo informal en tiempos de crisis. Política y Cultura, v. 22, p. 47-70, 2004.

NAROTZKY, S. Where have all the peasants gone?. Annual Review of Anthropology, v. 45, n. 1, p. 301-318, 2016.

OLIVER-SMITH, A. Anthropological research on hazards and disasters. Annual Review of Anthropology, v. 25, n. 1, p. 303-328, 1996.

OLIVER-SMITH, A. "What is a disaster?": anthropological perspectives on a persistent question. In: OLIVER-SMITH, A.; HOFFMAN, S. M. (ed.). The angry Earth. London: Routledge, 1999. p. 32-48.

PFAU-EFFINGER, B.; FLAQUER, L.; JENSEN, P. H. (ed.). Formal and informal work: the hidden work regime in Europe. New York: Routledge, 2010.

PIKETTY, T. Capital in the twenty-first century. Paris: Harvard University Press, 2014. 
PLOEG, J. D. van der. The peasantries of the twenty-first century: the commoditisation debate revisited. The Journal of Peasant Studies, v. 37, p. 1-30, 2010.

PLOEG, J. D. van der. The political economy of agroecology. The Journal of Peasant Studies, ahead of print, p. 1-24, 2020.

RABOSSI, F. Los caminos de la informalidad. Sociologia \& Antropologia, v. 9, n. 3, p. 797-818, 2019.

SHORE, C. La antropología y el estudio de la política pública: reflexiones sobre la "formulación" de las políticas. Antípoda: Revista de Antropología y Arqueología, v. 10, p. 21-49, 2010.

SOPER, R. From protecting peasant livelihoods to essentializing peasant agriculture: problematic trends in food sovereignty discourse. The Journal of Peasant Studies, v. 47, n. 2, p. 265-285, 2020.

STANDING, G. Por qué el precariado no es un "concepto espurio". Sociología del Trabajo, v. 82, p. 7-15, 2014.

VALENZUELA, H.; REYGADAS, L.; CRUCES, F. Mi trabajo es mi vida. La incrustación de los mundos de la vida y del trabajo en empresas españolas. Revista Española de Investigaciones Sociológicas, v. 150, n. 1, p. 191-206, 2015.

WHITSON, R. Beyond the crisis: economic globalization and informal work in urban Argentina. Journal of Latin American Geography, v. 6, n. 2, p. 121-136, 2007.

WOLF, E. Europa y la gente sin historia. México: Fondo de Cultura Económica, 1982.

Recebido: 31/07/2020 Aceito: 07/09/2020 | Received:7/31/2020 Accepted: 9/7/2020 